\title{
Intoxicação por Ipomoea asarifolia em ovinos e bovinos na llha de Marajó ${ }^{1}$
}

\author{
Fábio Py Tortelli², José Diomedes Barbosa ${ }^{3}$, Carlos Magno Chaves Oliveira ${ }^{3}$, \\ Marcos Dutra Duarte ${ }^{3}$, Valíria Duarte Cerqueira ${ }^{3}$, Carlos Alberto Oliveira ${ }^{3}$, \\ Franklin Riet Correa ${ }^{4}$ e Gabriela Riet Correa ${ }^{3^{*}}$
}

\begin{abstract}
Tortelli P.F., Barbosa J.D., Oliveira C.M.C., Dutra M.D., Cerqueira V.D., Oliveira C.A., Riet-Correa F. \& Riet-Correa G. 2008. [Poisoning by Ipomoea asarifolia in cattle and sheep in the Marajó Island, Northern Brazil.] Intoxicação por Ipomoea asarifolia em bovinos e ovinos na llha de Marajó. Pesquisa Veterinária Brasileira 28(12):622-626. Central de Diagnóstico Veterinário, Faculdade de Medicina Veterinária, Campus de Castanhal, Universidade Federal do Pará, Rua Maximino Porpino da Silva 1000, Pirapora, Castanhal, PA 68743-080, Brazil. E-mail: griet@ufpa.br

To determine plant poisonings occurring on Marajo Island, state of Pará, northern Brazil, 7 farms were visited. All farms had native pastures with low forage availability severely infected by Ipomoea asarifolia. In 5 farms poisoning in sheep by the plant was reported. The disease occurs mainly during the dry period and lambs are more frequently affected than adult sheep. Sheep with clinical signs were observed in 2 farms. In 4 establishments farmers reported the occurrence of $I$. asarifolia poisoning in cattle. In one farm affected cattle were observed during two visits, one during the dry season and another at the start of the raining season. Deaths are observed mainly in calves. In both species the poisoning occurs every year with variable morbidity and low mortality. One bovine and one sheep with severe clinical signs were euthanized and post-mortem examination was performed. No gross or histologic significant lesions were observed. In all farms buffaloes were raised together with cattle and sheep, and in 3 farms goats were also raised. None of the farmers reported the intoxication in these species.
\end{abstract}

INDEX TERMS: Cattle, Ipomoea asarifolia, sheep, toxic plants, tremogenic toxins.

RESUMO.- Para estudar as intoxicações por plantas que ocorrem na llha de Marajó foram visitadas 7 fazendas. Em todas as fazendas visitadas as pastagens eram constituídas de campo nativo, tinham pouco ou nenhuma disponibilidade de forragem e estavam severamente invadidas por

\footnotetext{
${ }^{1}$ Recebido em 27 de maio de 2008.

Aceito para publicação em 22 de julho de 2008

2 Programa de Pós-Graduação em Ciência Animal, Núcleo de Ciências Agrárias e Desenvolvimento Rural, Rua Augusto Corrêa 1, Campus Universitário do Guamá, Belém, PA 66075-110, Brazil. E-mail: pytortelli@yahoo.com.br

${ }^{3}$ Central de Diagnóstico Veterinário, Faculdade de Medicina Veterinária, Campus de Castanhal, Universidade Federal do Pará, Rua Maximino Porpino da Silva 1000, Pirapora, Castanhal, PA 68743-080, Brazil. *Autor para correspondência: griet@ufpa.br

${ }^{4}$ Hospital Veterinário, Centro de Saúde e Tecnologia Rural (CSTR), Universidade Federal de Campina Grande (UFCG), Campus de Patos, 58700-970 Patos, PB, Brasil. E-mail: franklin.riet@pq.cnpq.br
}

Ipomoea asarifolia. Nas 5 propriedades foi relatada a ocorrência da intoxicação por esta planta em ovinos. Animais jovens são mais afetados do que adultos. Em duas dessas propriedades foram observados ovinos com sinais clínicos. Em 4 fazendas, os proprietários relataram a ocorrência da intoxicação por $I$. asarifolia em bovinos e, em uma dessas, foram observados bovinos com sinais clínicos em duas visitas realizadas, uma no período seco e outra no início do período chuvoso. Em bovinos as mortes ocorrem principalmente quando são afetados bezerros. A doença é observada todos os anos, com morbidade variável e baixa mortalidade. Os sinais clínicos foram característicos de uma doença tremogênica. Um bovino e um ovino, que apresentavam sinais clínicos acentuados e foram eutanasiados e necropsiados, não apresentavam alterações macroscópicas nem histológicas de significação. Em todas as fazendas visitadas eram criados bubalinos juntamente com bovinos e/ou ovinos e em 3 propriedades eram criados capri- 
nos, mas nenhum proprietário relatou a ocorrência da intoxicação nestas duas espécies.

TERMOS DE INDEXAÇÃO: Bovinos, Ipomoea asarifolia, ovinos, plantas tóxicas, toxinas tremorgênicas.

\section{INTRODUÇÃO}

Na região Norte as intoxicações por plantas começaram a ser estudadas na década de 1970 definindo-se que Palicoureia marcgravii, Arrabidaea bilabiata e Arrabidaea japurensis são as três plantas mais importantes da região Amazônica. Outras duas plantas, Palicoureia juruana que ocorre em áreas restritas do Pará, Rondônia e Amazonas, e Palicoureia grandiflora que ocorre em Rondônia, têm uma distribuição limitada. Ocorrem, também, na região Amazônica, porem, com menor importância, Ipomoea carnea subsp. fistulosa, Ipomoea asarifolia, Lantana camara, Pteridium aquilinum, Ricinus comunis, Manihot spp, e Brachiaria spp. (Tokarnia et al. 2007). Em eqüinos são descritas as intoxicações por Bambusa vulgaris f. vulgaris (bambú) (Barbosa et al. 2006b) e por Brachiaria humidicola (Barbosa et al. 2006a).

$\mathrm{Na}$ llha de Marajó, embora exista grande quantidade de $I$. asarifolia e I. carnea subsp. fistulosa, não há relatos de intoxicação por essas plantas em caprinos, ovinos e bubalinos. A intoxicação por I. asarifolia foi observada nessa região somente em bovinos (Barbosa et al. 2005).

O objetivo do presente trabalho foi descrever a intoxicação por Ipomoea asarifolia em bovinos e ovinos na llha de Marajó.

\section{MATERIAL E MÉTODOS}

Foram visitadas 7 propriedades rurais na Ilha de Marajó, 6 localizadas no município de Cachoeira do Arari e uma no município de Souri, com histórico da ocorrência de doença tremorgênica. Em todas as propriedades eram criados búfalos, em duas havia também bovinos, em 5 havia ovinos e em 3 caprinos Durante as visitas foi realizado levantamento das plantas tóxicas existentes, estudo epidemiológico das intoxicações, avaliação clínica de animais intoxicados, necropsias de animais gravemente doentes e coleta de material para estudo histopatológico. Foram eutanasiados e necropsiados um ovino e um bovino. Durante a necropsia dos animais foram coletados fragmentos de todos os órgãos da cavidade abdominal e torácica e encéfalo. O encéfalo, após a fixação, foi cortado transversalmente, coletando-se fragmentos nas regiões do córtex parietal, temporal, occipital e frontal, núcleos da base, tálamo, mesencéfalo a altura do colículo
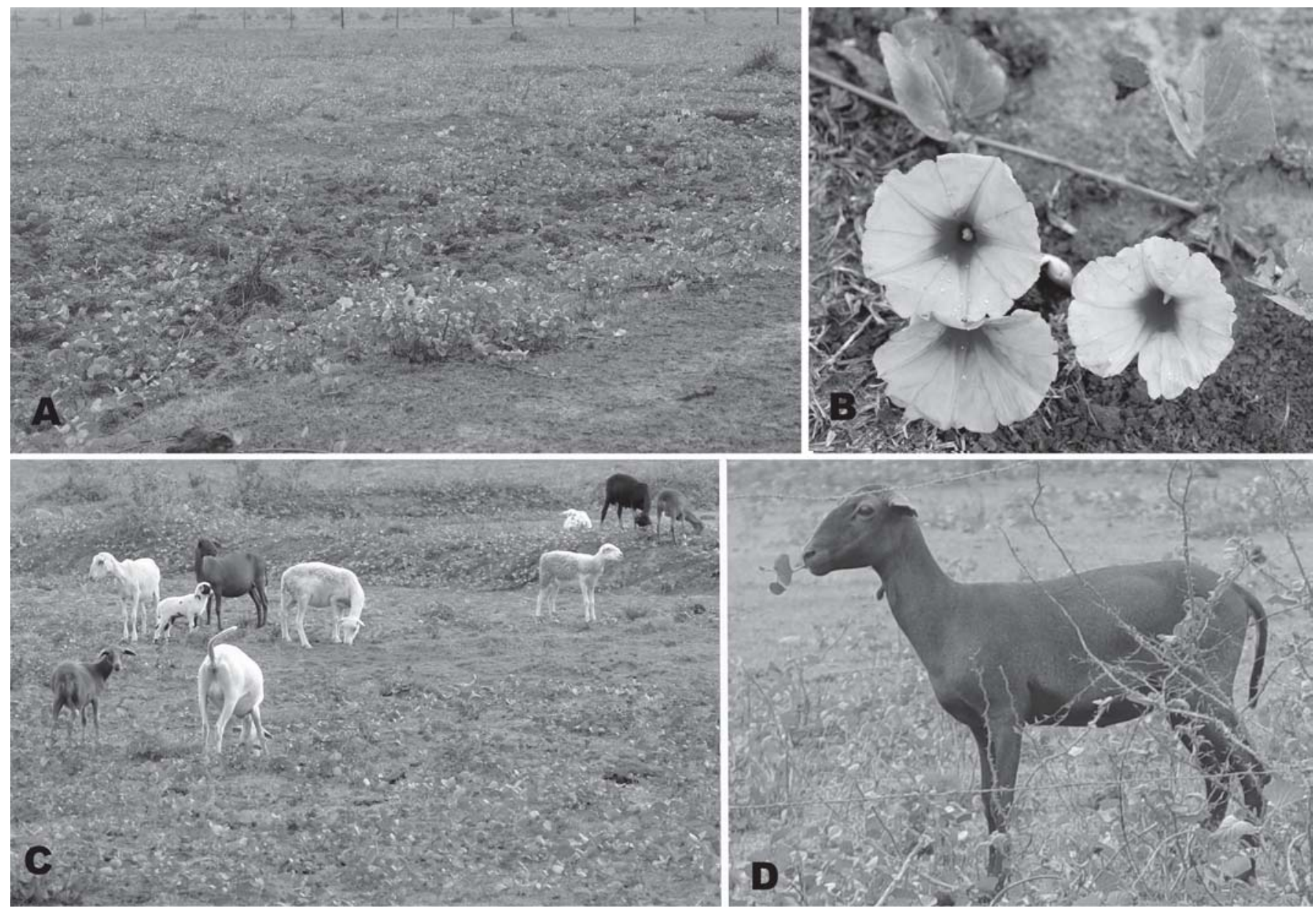

Fig.1. (A) Pasto severamente invadido pela "salsa", Ipomoea asarifolia, no município de Cachoeira do Arari. (B) Flores e folhas de I. asarifolia. (C) Ovinos pastejando em uma área severamente invadida por I. asarifolia. (D) Ovino ingerindo folhas de I. asarifolia. 
rostral, pedúnculos cerebelares, cerebelo, ponte, óbex e medula cervical.

\section{RESULTADOS}

Em todas as propriedades visitadas as pastagens eram constituídas de campo nativo, tinham pouco ou nenhuma disponibilidade de forragem e estavam severamente invadidas pela "salsa", Ipomoea asarifolia (Fig.1). O "canudo", Ipomoea carnea subsp. fistulosa, também foi observada nas pastagens, mas em menor quantidade.

Nas 5 propriedades onde eram criados ovinos foi relatada a ocorrência da intoxicação por I. asarifolia. Segundo os proprietários a doença ocorre principalmente durante o período seco e animais jovens são mais afetados do que adultos. Em duas dessas propriedades foram observados ovinos com tremores de intenção e incoordenação. Ao serem movimentados os sinais clínicos se acentuavam ocorrendo quedas em decúbito lateral com os membros rígidos em extensão (Fig.2). Um animal que apresentava sinais acentuados foi entanasiado e necropsiado; não se observaram alterações macroscópicas nem microscópicas de significação. Em uma proprie- dade a doença ocorria todos os anos em ovinos e bovinos, mas casos da intoxicação não foram mais observados em ovinos após a introdução de uma pastagem de Brachiaria humidicola para essa espécie.

Nas duas propriedades onde eram criados bovinos, e em outras duas propriedades, onde eram criados bovinos em anos anteriores, os proprietários relataram a ocorrência da intoxicação por I. asarifolia. A doença é observada todos os anos, com morbidade variável e baixa mortalidade, e as mortes ocorrem principalmente quando são afetados bezerros. Em uma das propriedades foram realizadas duas visitas, uma no período seco e outra no início do período chuvoso. Nas duas visitas foram observados bovinos com sinais clínicos nervosos caracterizados por abdução dos membros quando em estação, tremores de intenção, ataxia, hipermetria acentuada e quedas ao serem agitados (Fig.3). Um bovino que apresentava sinais clínicos acentuados foi eutanasiado e necropsiado; não se observaram alterações macroscópicas nem histológicas.

Em todas as propriedades visitadas eram criados bubalinos juntamente com bovinos e/ou ovinos, mas ne-
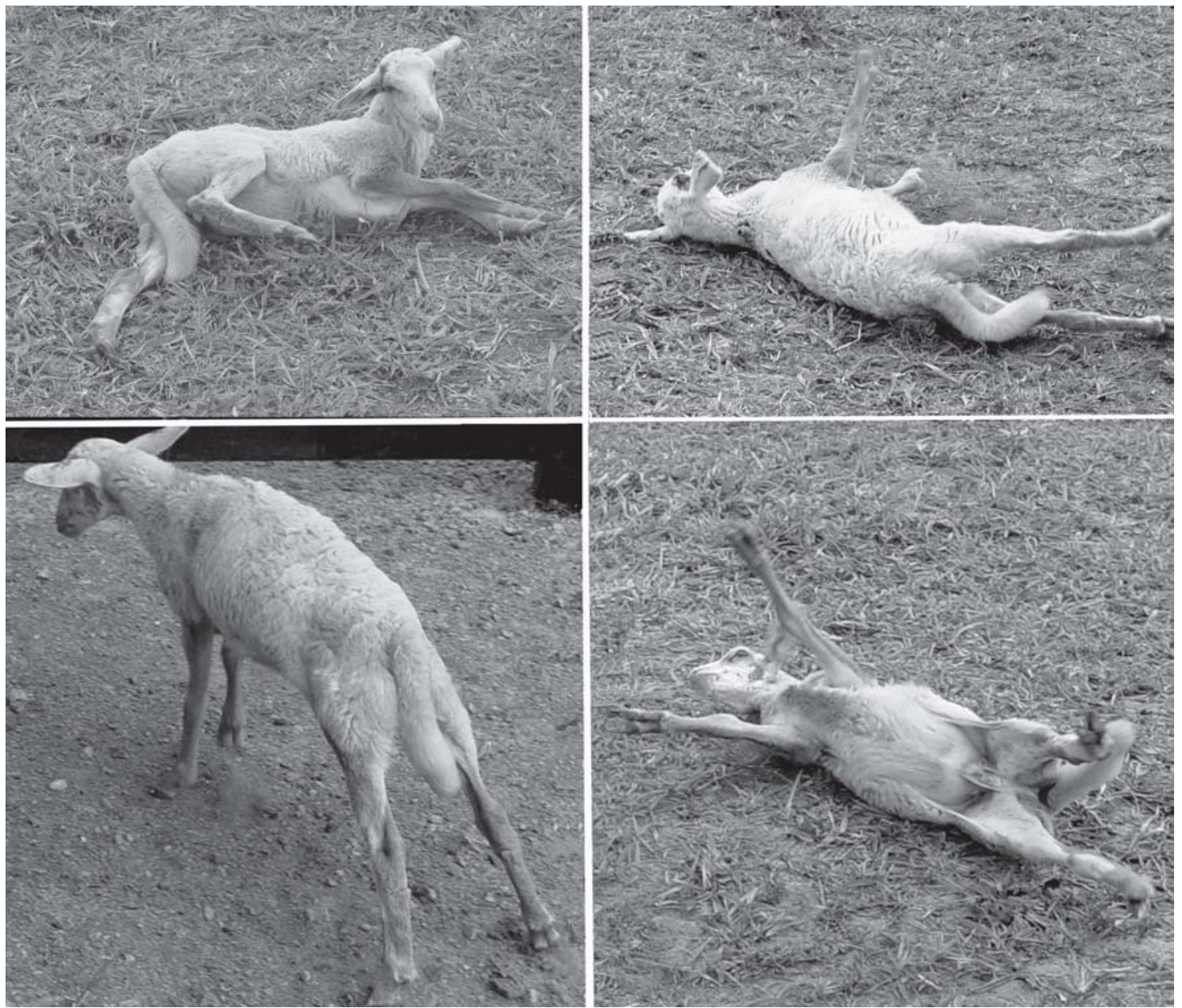

Fig.2. Ovino intoxicado por Ipomoea asarifolia, que apresentava tremores de intenção e ao ser movimentado os sinais clínicos se acentuaram apresentando membros rígidos em extensão e quedas. 

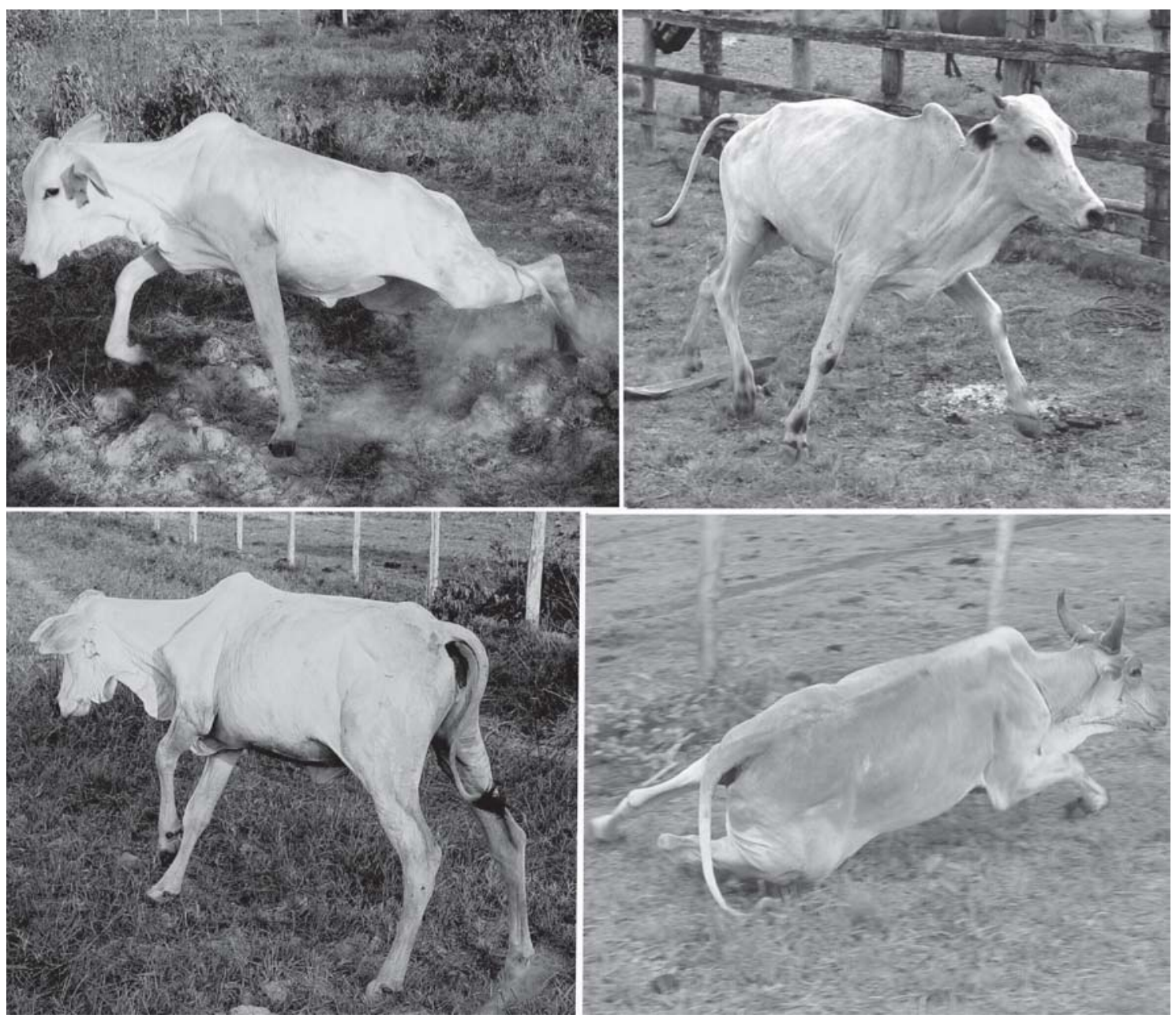

Fig.3. Bovinos intoxicados por Ipomoea asarifolia, que apresentavam tremores de intenção e ao serem movimentados apresentaram hipermetria, ataxia e quedas.

nhum proprietário relatou a ocorrência da intoxicação nessa espécie. A doença também não foi observada em caprinos, nas três propriedades que criavam esta espécie.

\section{DISCUSSÃO}

A presença de sinais clínicos característicos de uma doença tremorgênica, assim como ausência de lesões histológicas de significação, permite o diagnóstico de intoxicação por Ipomoea asarifolia, tanto em ovinos quanto em bovinos. Em caprinos e em bubalinos não foi constatada a doença; mas em caprinos foi diagnosticada, nestas mesmas fazendas, intoxicação por l. carnea subsp. fistulosa (Gabriela Riet-Correa, 2007, dados não publicados).

Essas informações confirmam que o ovino é a espécie mais afetada pela intoxicação por I. asarifolia e que entre os ovinos a faixa etária mais afetada é a dos cordeiros. Observações semelhantes são relatadas na região semi-árida do Nordeste (Riet-Correa et al. 2003, 2006, Guedes et al. 2007) onde também os bovinos são menos afetados do que os ovinos (Riet-Correa et al. 2003, 2006). $\mathrm{Na}$ llha de Marajó, apesar de que o maior número de ca- sos ocorreu em ovinos, é evidente que a doença é importante também em bovinos. Surtos de intoxicação por $I$. asarifolia em bovinos foram relatados anteriormente na Ilha de Marajó e a doença foi reproduzida experimentalmente (Barbosa et al. 2005). Chama a atenção, a ausência de casos da doença em caprinos e bubalinos, apesar de que experimentalmente estas duas espécies são também susceptíveis (Döbereiner et al. 1960, Medeiros et al. 2003, Barbosa et al. 2005) e a intoxicação espontânea tem sido descrita, também, em caprinos (Döbereiner et al. 1960, Riet-Correa et al. 2003, 2006). Em estudos comparativos os ovinos demonstraram ser um pouco mais sensíveis que os caprinos (Araújo et al. 2008) e os bovinos apresentam sensibilidade semelhante à dos bubalinos (Barbosa et al. 2005). Em relação à faixa etária foi demonstrado que ovinos jovens apresentam susceptibilidade semelhante à dos ovinos adultos (Araújo et al. 2008). Não deve ser descartada a possibilidade de intoxicação por I. asarifolia ocorra, também, em caprinos e bubalinos na llha de Marajó.

Os resultados deste trabalho mostram que a intoxicação por I. asarifolia é de extrema importância na região, 
visto a baixa disponibilidade de forragem na época seca, já que a maioria das pastagens é formada por campo nativo e que esta planta, compõe, na grande maioria dos casos, aproximadamente $80 \%$ da alimentação disponível para os animais.

Agradecimentos.- Este projeto foi financiado pelo programa Institutos do Milênio, CNPq (Proc.420012/2005-2).

\section{REFERÊNCIAS}

Araújo J.A.S., Riet-Correa F., Medeiros R.M.T., Soares M.P., Oliveira D.M. \& Carvalho F.K.L. 2008. Intoxicação experimental por Ipomoea asarifolia (Convolvulaceae) em caprinos e ovinos. Pesq. Vet. Bras. 28(10):488-494.

Barbosa J.D., Oliveira C.M.C., Tokarnia C.H. \& Peixoto P.V. $2006 a$. Fotossensibilização hepatógena em eqüinos pela ingestão de Brachiaria humidicola (Gramineae) no Estado do Pará. Pesq. Vet. Bras. 26(3):147-153

Barbosa J.D., Oliveira C.M.C., Duarte M.D., Correa G.R. \& Tokarnia C.H. 2006b. Poisoning of horses by bamboo, Bambusa vulgaris. J. Eq. Vet. Sci. 26:393-398.

Barbosa J.D., Oliveira C.M.C., Duarte M.D., Peixoto P.V. \& Tokarnia
C.H. 2005. Intoxicação experimental e natural por Ipomoea asarifolia (Convolvulaceae) em búfalos e outros ruminantes. Pesq. Vet. Bras. 25(4):231-234.

Döbereiner J., Tokarnia C.H. \& Canella C.F.C. 1960. Intoxicação experimental pela "salsa" (Ipomoea asarifolia R. et Schult.). Arqs Inst. Biol. Anim., Rio de J., 3:39-57.

Guedes K.M.R., Riet-Correa F., Dantas A.F.M., Simões S.V.D., Miranda Neto E.G., Nobre V.M.T. \& Medeiros R.M.T. 2007. Doenças do sistema nervoso central em caprinos e ovinos no semi-árido. Pesq. Vet. Bras. 27(1):29-38.

Medeiros R.M.T., Barbosa R.C., Riet-Correa F., Lima E.F., Tabosa I.M., Barros S.S., Gardner D.R. \& Molyneux R.J. 2003. Tremorgenic syndrome in goats caused by, Ipomoea asarifolia in northeastern Brazil. Toxicon 41:933-935.

Riet-Correa F., Tabosa I.M., Azevedo E.O., Medeiros R.M.T., Simões S.V.D., Dantas A.F.M., Alves C.J., Nobre V.M.T., Athayde A.C.R., Gomes A.A. \& Lima E.F. 2003. Doenças dos ruminantes e eqüinos no semi-árido da Paraíba. Semi-árido em Foco 1(1):58-60.

Riet-Correa F., Medeiros R.M.T. \& Dantas A.F.M. 2006. Plantas Tóxicas da Paraíba. UFCG/CSTR/HV, SEBRAE/PB, Patos. 58p.

Tokarnia C.H., Döbereiner J., Peixoto P.V., Barbosa J.D., Brito M.F. \& Silva M.F. 2007. Plantas Tóxicas da Amazônia. $2^{a}$ ed. INPA, Manaus. 96p. 